\title{
Post-testicular Sperm DNA Oxidation: What to Be Afraid of?
}

\author{
Joël R. Drevet
}

Normal embryo development, as well as the health of the progeny, is partly dependent on gamete nuclear integrity. If sperm DNA fragmentation that could be in part due to oxidative nuclear alterations is known to impact reproductive success, no one ever considers the impact of mild sperm DNA oxidative damage. To analyze this situation, we have developed mouse models that display some level of post-testicular sperm DNA oxidative damage. The data presented will first focus on why mammalian spermatozoa are susceptible to DNA oxidation, and where these oxidative alterations are located in the mouse sperm nucleus and chromosomes. Translation to the clinic will then be made with on the one hand the characterization of human sperm DNA susceptibility to oxidative damage and, on the other hand, the development of an assay allowing a reliable and accurate evaluation of oxidative DNA damage in human sperm samples. Data will also be presented showing how frequent this situation is in male patients having difficulty to conceive. In addition, preliminary data will show that besides base oxidative alterations the sperm epigenetic information (with a particular focus on sperm DNA methylation status and the small noncoding RNA sperm pool) may be affected by post-testicular oxidative stress.

\footnotetext{
J. R. Drevet (四)

Genetics Reproduction \& Development Laboratory (GReD), CNRS UMR6293-INSERM U1103-Université Clermont Auvergne (UCA), Clermont-Ferrand, France

e-mail: joel.drevet@uca.fr 